\section{Evolução do estado nutricional na doença de Gaucher tipo I em tratamento com reposição enzimática - relato de dois casos}

\section{The evolution of nutritional status in type I Gaucher's disease treated with enzyme replacement - two case histories}

Andréa Vieira Pereira Coradine 1

Mara Albonei Dudeque Pianovski 2
1 Programa de Pós-graduação em Saúde da Criança e do Adolescente. Universidade Federal do Paraná. Rua General Carneiro 181, $14^{\circ}$ andar. Curitiba, PR, Brasil. E-mail: andrea_ufpr@yahoo.com.br

2 Serviço de Oncologia Pediátrica. Hospital de Clínicas. Universidade Federal do Paraná. Curitiba, PR, Brasil.

Resumo

Introdução: a doença de Gaucher é um erro do metabolismo enzimático que leva ao acúmulo de glicocerebrosideo nas células, o que caracteriza os sinais e sintomas da doença. No momento do diagnóstico, além de outros sinais e sintomas, é observado retardo no crescimento em crianças e adolescentes. $O$ tratamento é realizado por meio de reposição enzimática, que pode ocasionar ganho de peso no paciente pela diminuição do metabolismo energético.

Descrição: dois irmãos com diagnóstico de doença de Gaucher tipo I foram avaliados antes de iniciarem a reposição enzimática e depois a cada 2 meses de tratamento, por um periodo de 6 meses. A composição corporal foi avaliada por impedância bioelétrica, que avaliou a quantidade de massa livre de gordura e massa de gordura; o consumo energético e a relação de macronutrientes foram avaliados por registro alimentar de 3 dias.

Discussão: os dois pacientes apresentavam baixa estatura para idade ao diagnóstico e tiveram aumento de massa de gordura durante o tratamento, sendo que um paciente também apresentou aumento da massa livre de gordura. O consumo energético e a relação de macronutrientes mantiveram-se semelhantes durante todo o periodo de acompanhamento para ambos os pacientes.

Palavras-chave Doença de Gaucher, Estado nutricional, Consumo alimentar, Peso corporal 


\section{Introdução}

A doença de Gaucher é um erro inato do metabolismo com mutação no gene que codifica a enzima beta-glicosidase ácida ou beta-glicocerebrosidase. Esta enzima atua no metabolismo dos esfingolipídeos e sua deficiência leva ao acúmulo progressivo do seu substrato, o glicocerebrosídeo, um glicolipídio. Este acúmulo acontece nos macrófagos, que recebem o nome de células de Gaucher e são depositados principalmente no baço, fígado, medula óssea e pulmão, o que resulta nas manifestações clínicas da doença que incluem: hepatoesplenomegalia, anemia, trombocitopenia, atraso do crescimento e doença esquelética. 1,2

Dependendo do grau de deficiência da enzima e da quantidade de substrato acumulado, três fenótipos distintos são descritos: tipo I ou forma não neuropática (ausência de doença primária no sistema nervoso central - SNC), tipo II ou forma neuropática aguda (comprometimento grave do SNC na infância) e tipo III ou forma neuropática crônica (comprometimento do SNC que permite sobrevida além da primeira infância). A incidência da doença de Gaucher é de 1 para cada grupo de 40.000 a 60.000 nascidos vivos na população geral, sendo o tipo I o mais comum ( $95 \%$ dos casos). 3

Na doença de Gaucher tipo I, a idade de aparecimento dos sintomas e intensidade de sua manifestação são variáveis. Apesar de tradicionalmente o tipo I ser referido como "tipo adulto", 66\% dos casos se manifestam na infância. ${ }^{1}$ Quando identificada na infância, a doença é mais grave e há alto risco de complicações mórbidas. Há acentuado catabolismo que, associado à esplenomegalia, desencadeia rápida plenitude gástrica pós-prandial levando a retardo no crescimento. ${ }^{1}$

O tratamento é feito pela reposição enzimática (TRE) que diminui o acúmulo do substrato nas células e melhora a qualidade de vida. Observa-se que, durante o tratamento, os pacientes apresentam ganho de peso uma vez que o medicamento diminui o gasto metabólico que se encontrava aumentado antes da reposição enzimática. ${ }^{2}$

De acordo com o registro de doença de Gaucher, ${ }^{2}$ existem aproximadamente 5000 pacientes com diagnóstico de doença de Gaucher tipo I no mundo, sendo quase 500 no Brasil. Devido a raridade da doença, ao pequeno número de pacientes no Brasil com o diagnóstico e a possibilidade de avaliação antes do início do tratamento, houve o interesse em relatar o caso de dois irmãos com doença de Gaucher tipo I durante o período de seis meses de tratamento com terapia de reposição enzimática com
Imiglucerase ${ }^{\circledR}$, uma enzima obtida pela tecnologia de DNA recombinante usando células de ovário de Hamster. Os pacientes estão em tratamento no ambulatório de hemato-oncopediatria do Hospital de Clínicas do Paraná em Curitiba.

\section{Descrição}

Dois irmãos, raça branca, um do gênero feminino, com 13 anos (paciente 1) e outro do gênero masculino com 10 anos (paciente 2), tiveram diagnóstico de doença de Gaucher tipo I em maio de 2012 baseado nos seguintes sintomas: anemia persistente, plaquetopenia e esplenomegalia; não apresentavam comprometimento neurológico. Foram tratados com Imiglucerase ${ }^{\circledR}$ na dose de $30 \mathrm{U} / \mathrm{kg}$, em administração endovenosa em infusão de 2 horas a cada 15 dias.

No momento do diagnóstico os pacientes apresentavam os seguintes sintomas:

- Paciente 1: anemia, plaquetopenia, hepatoesplenomegalia, lesão lítica no fêmur, estatura para idade $<$ percentil 3 e estatura alvo de acordo com estatura dos pais $<$ percentil 50;

- Paciente 2: plaquetopenia, hepatoesplenomegalia, estatura para idade $<$ percentil 25 e estatura alvo de acordo com estatura dos pais $<$ percentil 50 .

Após 6 meses de TRE os irmãos apresentavam os seguintes sintomas:

- Paciente 1: hepatoesplenomegalia, lesão lítica no fêmur, baixa estatura para idade e estatura alvo de acordo com estatura dos pais $<$ percentil 50;

- Paciente 2: hepatoesplenomegalia, baixa estatura para idade e estatura alvo de acordo com estatura dos pais $<$ percentil 50 .

Utilizando-se o escore PGS3 (Pediatric Gaucher Severity Scoring System) estabelecido por Kallish e Kaplan, ${ }^{4}$ para avaliar a gravidade da doença nos pacientes, estes receberam pontuação de 8,25 e 5,16 para o paciente 1 e 2 respectivamente, sendo considerados com gravidade média e baixa.

A avaliação do estado nutricional foi realizada antes de iniciarem TRE (mês 0 ) e nos meses 2, 4 e 6 de tratamento.

Foram avaliados os seguintes parâmetros: a) índice de massa corporal (IMC); b) estatura/idade; c) massa livre de gordura (MLG) e d) massa de gordura (MG).

O cálculo do IMC foi feito usando a fórmula: $\mathrm{IMC}=$ Peso $(\mathrm{kg}) /$ Estatura (metro) ${ }^{2}$. O escore $\mathrm{Z}$ foi obtido através do software WHO Anthro Plus ${ }^{\circledR} 2009$ e classificado pelo índice IMC para Idade de acordo com a Organização Mundial da Saúde (OMS) de 2006 e 2007.5 
A MLG e MG foram avaliadas com impedância bioelétrica (BIA) utilizando-se o aparelho Maltron BioScan MSR-916 ${ }^{\circledR}$. Para minimizar erros entre as avaliações foi padronizada a realização da BIA sempre após jejum mínimo de 4 horas, no horário matutino, espaçamento mínimo entre os eletrodos de 5 centímetros e repouso em decúbito dorsal por, no mínimo, 10 minutos. Os pacientes e seus cuidadores foram instruídos de que os mesmos não deveriam ingerir café, chocolate e bebidas cafeinadas no dia anterior ao exame e nem praticar atividade física intensa nas 24 horas anteriores. Para o cálculo da MLG, valores de resistência e reactância, obtidos pela BIA, foram utilizados nas fórmulas estabelecidas por Houtkooper et al. ${ }^{6}$ de acordo com o sexo: MLG meninas $=0,61\left(\mathrm{~A}^{2} / \mathrm{R}\right)+0,25(\mathrm{P})+1,31 \mathrm{e}$ MLG meninos $=0,61\left(\mathrm{~A}^{2} / \mathrm{R}\right)+0,25(\mathrm{P})+1,31$ (onde: $\mathrm{P}=$ peso em $\mathrm{kg} ; \mathrm{A}=$ altura em $\mathrm{cm} ; \mathrm{R}=$ resistência). Para a obtenção do valor da $M G$ foi feita a subtração da MLG do peso corporal.

A avaliação do consumo alimentar foi realizada antes do início do tratamento, no $2^{\circ}, 4^{\circ}$ e $6^{\circ}$ mês de avaliação, por meio do registro alimentar de três dias. Foi avaliado o consumo energético e de macronutrientes através do programa Avanutri ${ }^{\circledR}$.

A pesquisa foi aprovada pelo Comitê de Ética em Pesquisa em Seres Humanos do Hospital de Clínicas da Universidade Federal do Paraná sob o parecer $n^{\circ}$ 051.2013.

\section{Discussão}

Comparando a avaliação basal com a realizada aos seis meses de tratamento foi possível observar que:

- a paciente 1 teve aumento de $2,4 \mathrm{Kg}$, sendo que a $\mathrm{MG}$ aumentou 3,9 $\mathrm{kg}$ e a MLG diminuiu $1,5 \mathrm{~kg}$;

- o paciente 2 teve aumento de $3,4 \mathrm{Kg}$, sendo 2,8 $\mathrm{kg}$ em MG e $620 \mathrm{~g}$ em MLG;

De acordo com a curva de crescimento da OMS, 6 na avaliação pré tratamento, a paciente 1 apresentava escore $\mathrm{Z}$ de estatura para idade e de IMC para idade iguais a $-2,09$ e $-1,15$, respectivamente, sendo que ao completar 6 meses de TRE, esses valores se alteraram para $-2,03$ e $-0,97$. Os valores de escore $Z$ pré tratamento do paciente 2 foram $-1,08$ e $-0,70$ e, ao $6^{\circ}$ mês de tratamento, $-1,19$ e $-0,03$ para estatura para idade e IMC para idade, respectivamente.

A Tabela 1 apresenta os valores da avaliação nutricional dos pacientes.

Como o consumo energético e a relação de macronutrientes não apresentaram variação clínica significante durante o período de acompanhamento (Tabela 2), a variação de peso e MG nos pacientes parece ser resultado do tratamento com
Imiglucerase ${ }^{\circledR}$.

A ação desta enzima na regulação do metabolismo ainda não é plenamente conhecida. Alguns autores avaliaram o gasto energético de pacientes com doença de Gaucher tipo I por calorimetria indireta e observaram hipermetabolismo com gasto energético variando entre 24 e $44 \%$ acima do consumo considerado normal, sendo que à avaliação pós tratamento e/ou nos pacientes em tratamento de reposição enzimática o gasto energético foi diminuído mas não normalizado. $7-9$

Analisando a necessidade energética pela fórmula preconizada pela Food and Agriculture Organization of the United Nations / World Health Organization (FAO/WHO) ${ }^{10}$ observou-se que para a paciente 1 a média de necessidade durante o acompanhamento foi de $1845 \mathrm{Kcal}$ ao dia enquanto que para o paciente 2 este valor foi de 1814 Kcal. A análise detalhada do consumo alimentar realizada para os dois pacientes aqui descritos não identificou aumento de ingestão calórica (de 1803,09 Kcal na primeira avaliação para $1798,34 \mathrm{kcal}$ na última para paciente 1 e de $1162,90 \mathrm{Kcal}$ para 1398,95 no paciente 2 no mesmo intervalo), sendo que o consumo energético dos pacientes estava abaixo do que seria considerado ideal em todos os momentos de avaliação. Pelos resultados aqui apresentados, questiona-se se o próprio tratamento com Imiglucerase ${ }^{\circledR}$ pode levar ao ganho de peso. Assim, conhecer as vias metabólicas pelas quais ocorre a ação da enzima sobre o metabolismo é fundamental para se afirmar tal questionamento, e então auxiliar no estabelecimento da conduta nutricional adequada para essa população de pacientes. Para isso, mais estudos precisam ser realizados para compreender a ação da reposição enzimática no metabolismo dos pacientes.

Como a avaliação precisa do gasto energético é difícil de ser realizada na prática clínica, o acompanhamento nutricional em crianças com doença de Gaucher é essencial para garantir a adequada composição corporal evitando-se obesidade, e promovendo o crescimento ideal, segundo seu potencial hereditário. Este acompanhamento deve ser frequente e incluir análise da composição corporal e do consumo alimentar para auxiliar no discernimento da conduta nutricional. 
Tabela 1

Avaliação nutricional dos pacientes antes do tratamento (mês 0 ) e a cada dois meses no período de seis meses de tratamento (mês 2, 4 e 6).

\begin{tabular}{|c|c|c|c|c|c|c|c|c|c|c|}
\hline & \multicolumn{2}{|c|}{ Peso (kg) } & \multicolumn{2}{|c|}{ Estatura (m) } & \multicolumn{2}{|c|}{ IMC $\left(\mathrm{kg} / \mathrm{m}^{2}\right)$} & \multicolumn{2}{|c|}{ MLG (kg) } & \multicolumn{2}{|c|}{ MG (kg) } \\
\hline & $1 *$ & $2 * *$ & $1 *$ & $2 * *$ & $1^{*}$ & $2 * *$ & $1 *$ & $2 * *$ & $1 *$ & $2 * *$ \\
\hline Mês 0 & 34,6 & 28,0 & 1,44 & 1,34 & 16,69 & 15,59 & 30,53 & 24,66 & 4,07 & 3,34 \\
\hline Mês 2 & 34,8 & 28,6 & 1,46 & 1,35 & 16,33 & 15,69 & 28,22 & 23,47 & 6,58 & 5,13 \\
\hline Mês 4 & 36,1 & 29,3 & 1,46 & 1,36 & 16,71 & 15,84 & 29,07 & 24,10 & 7,03 & 5,20 \\
\hline Mês 6 & 37,0 & 31,4 & 1,46 & 1,36 & 17,36 & 16,98 & 29,02 & 25,28 & 7,98 & 6,12 \\
\hline
\end{tabular}

$\mathrm{IMC}=$ índice de massa corporal; $\mathrm{MLG}=$ massa livre de gordura; $M G=$ massa de gordura; 1 * = paciente sexo feminino; $2^{* *}=$ paciente sexo masculino.

Tabela 2

Avaliação do consumo alimentar dos pacientes antes do tratamento (mês 0 ) e a cada dois meses no período de seis meses de tratamento (mês 2,4 e 6).

\begin{tabular}{|c|c|c|c|c|c|c|c|c|c|c|}
\hline & \multicolumn{2}{|c|}{ Kcal } & \multicolumn{2}{|c|}{$\mathrm{CHO}(\%)$} & \multicolumn{2}{|c|}{ PTN (\%) } & \multicolumn{2}{|c|}{ LIP (\%) } & \multicolumn{2}{|c|}{ Fibras (g) } \\
\hline & 1 * & $2 * *$ & 1 * & $2 * *$ & 1 * & $2 * *$ & 1 * & $2 * *$ & 1 * & $2 * *$ \\
\hline Mês 0 & 1803,09 & 1162,90 & 55,85 & 64,30 & 14,46 & 14,97 & 29,69 & 20,73 & 16,16 & 10,72 \\
\hline Mês 2 & 1754,87 & 1342,80 & 46,82 & 51,38 & 14,16 & 13,97 & 39,02 & 34,65 & 14,78 & 12,35 \\
\hline Mês 4 & 1842,38 & 1302,68 & 53,32 & 60,17 & 12,33 & 15,76 & 34,35 & 24,07 & 13,45 & 11,36 \\
\hline Mês 6 & 1798,34 & 1398,95 & 54,98 & 55,45 & 13,78 & 15,84 & 31,24 & 28,71 & 16,23 & 13,21 \\
\hline
\end{tabular}

$\mathrm{Kcal}=$ quilocaloria $\mathrm{CHO}=$ carboidrato; $\mathrm{PTN}=$ proteína; $\mathrm{LIP}=$ lipídeo; $1 *$ paciente sexo feminino; $2 * *=$ paciente sexo masculino.

\section{Referências}

1. Grabowski GA, Andria G, Baldellou A, Campbell PE, Charrow J, Cohen IJ, Harris CM, Kaplan P, Mengel E, Pocovi M, Vellodi A. Pediatric non-neuronopathic Gaucher disease: presentation, diagnosis and assessment. Consensus statements. Eur J Pediatr. 2004; 163 (2): 58-66.

2. Sobreira EAP, Bruniera P. Avaliação de dois anos de tratamento da doença de Gaucher tipo 1 com terapia de reposição enzimática em pacientes do estado de São Paulo, Brasil. Rev. Bras. Hematol Hemoter. 2008; 30: 193-201.

3. Gaucher Registry - International Collaborative Gaucher Group (ICGG). Disponível em: www.gaucherregistry.com

4. Kallish S, Kaplan P. A disease severity scoring system for children with type 1 Gaucher disease. Eur J Pediatr. 2013; 172: 39-43.

5. Onis M, Onyango AW, Borghi E, Siyam A, Nishida C, Siekmann J. Development of a WHO growth reference for school-aged children and adolescents. Bull World Health Organ. 2007; 85: 660-7.

Recebido em 4 de junho de 2014

Versão final apresentada em 11 de novembro de 2014

Aprovado em 16 de março de 2015
6. Houtkooper LB, Going SB, Lohman TG, Roche AF, Van Loan M. Bioelectrical impedance estimation of fat-free body mass in children and yuoth: a cross-validation study. $\mathrm{J}$ Appl Physiol. 1992; 72 (1): 366-73.

7. Corssmitt EPM, Hollak CE, Endert E, Van Oers MH, Sauerwein HP, Romijn JA. Increased basal glucose production in type 1 Gaucher's disease. J Clin Endocrinol Metab. 1995; 80: 2653-7.

8. Hollak CE, Corssmit EP, Aerts JM, Endert E, Sauerwein HP, Romijn JA, Van Oers MH. Differential effects of enzyme supplementation therapy on manifestations of type 1 Gaucher disease. Am J Med. 1997; 103: 185-91.

9. Langeveld M, Endert E, Wiersinga WM, Aerts JM, Hollak CE. Hypermetabolism in Gaucher disease type I is not associated with altered thyroid hormone levels. J Inherit Metab Dis. 2007; 30: 985 .

10. Food and Nutrition Board, National Research Council, National Academy of Sciences. Recommended Dietary Allowances, 10a ed. Washingtoon, DC: Nation al Academy Press; 1989. 